\title{
Similarity-based interference and relative clauses in second language processing
}

Article

Published Version

Creative Commons: Attribution 4.0 (CC-BY)

Open Access

Cunnings, I. ORCID: https://orcid.org/0000-0002-5318-0186 and Fujita, H. (2021) Similarity-based interference and relative clauses in second language processing. Second Language Research. ISSN 1477-0326 doi:

https://doi.org/10.1177/02676583211063534 Available at https://centaur.reading.ac.uk/101942/

It is advisable to refer to the publisher's version if you intend to cite from the work. See Guidance on citing.

To link to this article DOI: http://dx.doi.org/10.1177/02676583211063534

Publisher: Sage

All outputs in CentAUR are protected by Intellectual Property Rights law, including copyright law. Copyright and IPR is retained by the creators or other copyright holders. Terms and conditions for use of this material are defined in the End User Agreement.

\section{www.reading.ac.uk/centaur}

\section{CentAUR}

Central Archive at the University of Reading 
Reading's research outputs online 


\section{Similarity-based interference and relative clauses in second language processing}

Second Language Research

(C) The Author(s) 2021

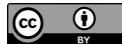

Article reuse guidelines: sagepub.com/journals-permissions DOI: I0.II77/026765832II063534

journals.sagepub.com/home/slr

@SAGE

\title{
lan Cunnings $(\mathbb{D}$ and Hiroki Fujita $(\mathbb{D}$
}

University of Reading, UK

\begin{abstract}
Relative clauses have long been examined in research on first (LI) and second (L2) language acquisition and processing, and a large body of research has shown that object relative clauses (e.g. 'The boy that the girl saw') are more difficult to process than subject relative clauses (e.g. 'The boy that saw the girl'). Although there are different accounts of this finding, memorybased factors have been argued to play a role in explaining the object relative disadvantage. Evidence of memory-based factors in relative clause processing comes from studies indicating that representational similarity influences the difficulty associated with object relatives as a result of a phenomenon known as similarity-based interference. Although similarity-based interference has been well studied in LI processing, less is known about how it influences L2 processing. We report two studies - an eye-tracking experiment and a comprehension task - investigating interference in the comprehension of relative clauses in LI and L2 readers. Our results indicated similarity-based interference in the processing of object relative clauses in both LI and L2 readers, with no significant differences in the size of interference effects between the two groups. These results highlight the importance of considering memory-based factors when examining L2 processing.
\end{abstract}

\section{Keywords}

eye-tracking, relative clauses, second language processing, similarity-based interference

\section{Introduction}

Relative clauses (RCs) have been widely examined in different subfields of linguistics, and a long line of research in psycholinguistics has demonstrated that object RCs like (1b), where the sentence subject ('the boy') is interpreted as the object of the RC verb 'saw', are more difficult to process than subject RCs like (1a) in English (for review, see

\footnotetext{
Corresponding author:

lan Cunnings, School of Psychology and Clinical Language Sciences, The University of Reading, Whiteknights Campus, Reading, RG6 7BE, UK

Email: i.cunnings@reading.ac.uk
} 
Gordon and Lowder, 2012; Lau and Tanaka, 2021). RCs have long been examined in research in second language (L2) acquisition (e.g. Doughty, 1991; Eckman et al., 1988; Gas, 1979) and, more recently, L2 processing (e.g. Baek, 2012; Jackson and Roberts, 2010; Xia et al., 2020). The asymmetry between subject and object RCs provides a good testing ground to inform debate about the nature of L2 acquisition and processing.

(1) a. The boy that saw the girl yesterday afternoon walked home.

b. The boy that the girl saw yesterday afternoon walked home.

Although there are various different accounts of the difficulty associated with object $\mathrm{RCs}$, the role that memory-based factors play in object RC processing has been highlighted in a number of studies (e.g. Gibson, 1998; Gordon and Lowder, 2012; Grodner and Gibson, 2005; Lewis et al., 2006; Lewis and Vasishth, 2005). Such accounts explain the object RC difficulty at least in part due to object RCs placing increased memory demands on language processing than subject RCs. That memory plays a role in explaining the object RC disadvantage is demonstrated by a series of studies reported by Gordon et al. (2001, 2004, 2006), who found that similarity-based interference influences the object RC disadvantage. That is, object RCs are particularly difficult to process when the two nouns are similar along a particular dimension. For example, object RCs that contain two common nouns (e.g. 'the boy' and 'the girl') are more difficult to process than object RCs containing one common noun and a proper name (e.g. 'the boy' and 'Susan').

To date, how similarity-based interference affects subject and object RCs in L2 processing has not been systematically examined. This lack of research in L2 processing motivated the current study. We report two experiments, an eye-tracking during reading task and an offline comprehension study, that examined how similarity-based interference affects subject and object RCs in first language (L1) English speakers and L2 English speakers from a variety of different L1 backgrounds. Below, we begin with discussion of research on RCs in L1 processing, before discussing the existing L2 processing literature.

\section{Relative clauses and similarity-based interference in $\mathrm{LI}$ processing}

A number of different accounts have been proposed to explain the object RC disadvantage. In standard linguistic theory, RC interpretation involves linking the sentence subject with an empty 'gap' position inside the RC. In subject RCs the gap appears in subject position ('The boy that _ saw the girl') while in object RCs it is the object of the RC verb ('The boy that the girl saw _'). The active-filler strategy (Frazier and Clifton, 1989) predicts that the sentence subject is always initially associated with the subject gap position during incremental processing, and as such explains processing difficulty in object RCs as resulting from reanalysis of this initial interpretation. On the other hand, expectation-based accounts (e.g. Levy, 2008) claim that object RCs are difficult mainly because they are less frequent than subject RCs, such that a fragment such as 'The boy that ...' is initially most likely to be interpreted as a subject RC because this is more frequent (for evidence of experience-based factors influencing RC processing, see e.g. Gennari and MacDonald, 2008; Reali and Christiansen, 2007; Roland et al., 2007; Wells 
et al., 2009). Memory-based accounts (Gibson, 1998; Grodner and Gibson, 2005; Lewis and Vasishth, 2005) predict difficulty in object RCs because in object RCs, but not subject RCs, the sentence subject needs to be maintained in memory until it is assigned a thematic role by the non-adjacent RC verb. Cue-based models (Lewis et al., 2006; Lewis and Vasishth, 2005) for example predict that memory retrieval of the sentence subject as the object of the RC verb causes difficulty during processing. That is, the verb 'saw' in (1b) triggers retrieval of the sentence subject ('the boy') from memory, but the linearly closer RC subject ('the girl') may interfere in this retrieval. It is likely that the difficulty associated with object RCs in English is a conflation of these multiple different demands during incremental processing, such that both expectation-based and memory-based factors influence RC processing (Staub, 2010; Staub et al., 2017).

The extent to which object RCs cause difficulty during processing of the RC itself and also the subsequent matrix verb ('walked') in sentences like (1b) in comparison to (1a) has also been debated. Cue-based models (e.g. Lewis and Vasishth, 2005; Van Dyke, 2007), for instance, would predict that in addition to difficulty during processing of the $\mathrm{RC}$ itself, the matrix verb in sentences containing object RCs should be more difficult to process than the corresponding matrix verb in sentences containing subject RCs. At 'walked' in (1a/b), the sentence subject ('the boy') needs to be retrieved and interpreted as the matrix verb subject. Cue-based models predict that memory retrieval during sentence processing involves matching a set of retrieval cues against all items in memory in parallel, with the item in the sentence that provides the best match being subsequently retrieved. Difficulty arises if multiple items match a set of retrieval cues. Given that the matrix verb requires a subject, cue-based models predict that retrieval at the matrix verb will include a cue for [+SUBJECT]. In object RCs like (1b), the intervening noun ("the girl') matches this cue (as subject of the RC), while in subject RCs like (1a) it does not. As such, the fact that (1b) contains two subjects should lead to similarity-based interference, and longer reading times, at the matrix verb in (1b) than (1a). This type of interference is called similarity-based retrieval interference because it results from the match between a set of cues utilized during retrieval and the items within a sentence. While a number of studies have found evidence of longer reading times at the matrix verb following object rather than subject RCs (Gordon et al., 2001, 2004, 2006; Johnson et al., 2011; King and Just, 1991; Lowder and Gordon, 2012, 2014; Traxler et al., 2002, experiments 1 and 3; Traxler et al., 2005), some have not (Staub, 2010; Traxler et al., 2002, experiment 2). Note that in all the cited studies that found longer reading times at the matrix verb following object than subject RCs, the matrix verb appeared immediately after the RC. Staub et al. (2017) demonstrated that difficulty at the matrix verb in sentences containing object RCs in part results from the fact that the matrix verb immediately follows the RC verb in object, but not subject, RCs. They found that when additional material was included as spillover between the RC verb and matrix verb (e.g. The boy that the girl saw in school walked home), there were no significant differences in reading times at the matrix verb as a result of RC type (see also Grodner and Gibson, 2005). These results suggest that previous results are confounded with spillover between the RC and matrix verb. Staub et al. took their findings as evidence against retrieval interference causing difficulty at the matrix verb. 
Evidence for the role of memory-based factors in the processing of object RCs is also provided by Gordon et al. (2001, 2004, 2006). Gordon et al. (2006) monitored participants' eye-movements as they read sentences like (2). They compared sentences containing subject RCs, as in (2a), and object RCs, as in (2b), and additionally manipulated the similarity of the sentence subject and RC noun, to be either two common nouns ('the banker'/‘the barber') or a common noun and a proper name ('the banker'/‘Sophie').

(2) a. The banker that praised the barber/Sophie climbed the mountain just outside of town.

b. The banker that the barber/Sophie praised climbed the mountain just outside of town.

At the RC region ('that praised the barber/Sophie', 'that the barber/Sophie praised'), Gordon et al. found an RC type by noun similarity interaction, such that the usual object $\mathrm{RC}$ disadvantage was observed in sentences where the two nouns were similar, that is two common names, but was significantly reduced, or eliminated, when the sentence subject and RC nouns were dissimilar. This pattern was also observed at the matrix verb, though as the object RC verb and matrix verb were adjacent, it is difficult to be certain whether this indexes difficulty at the matrix verb itself, or spillover from the RC region. Gordon et al. (2001) also reported similar effects in self-paced reading (see also Gordon et al., 2004), and additionally found a significant $\mathrm{RC}$ type by noun similarity interaction in comprehension accuracy in addition to processing times during reading. Participants in Gordon et al. (2001) answered true/false statements about the interpretation of the RC verb and matrix verb, and accuracy was high for all conditions except the object RC condition with two similar nouns. Gordon et al. (2006), however, did not replicate this interaction in comprehension accuracy. In sum, Gordon et al. (2001, 2004, 2006) interpreted these results as indexing similarity-based interference during processing. Specifically, they argued that similarity between the two nouns leads to less distinct representations in memory.

The results reported by Gordon et al. are typically described to be a form of encoding interference. This is different to the retrieval interference discussed previously. Retrieval interference occurs when an item in a sentence partially matches a set of retrieval cues. Encoding interference on the other hand cannot reasonably be explained in terms of potential cues at retrieval. There is no clear way, for example, why verbs would cue retrieval of a particular type of noun. Instead, interference here appears to arise solely because items in a sentence are similar along a particular dimension. This similarity leads to items being encoded in memory with less distinct representations, which leads to processing disruption.

Note that similarity-based encoding and retrieval interference are concepts that are in ways similar to work in theoretical linguistics on relativized minimality (Rizzi, 1990, 2018). Relativized minimality was advanced as a syntactic locality constraint on linguistic dependencies and predicts that a dependency between two constituents (X and Y) should be disrupted when a constituent $(\mathrm{Z}$ ) intervenes that c-commands $\mathrm{Y}$ (but not $\mathrm{X}$ ). The most recent instantiation of the theory predicts differing degrees of disruption based on the degree of featural match between constituents (Belletti and Rizzi, 2013; Rizzi, 2018). We do not attempt to tease these two accounts here, though note that similarity-based interference predicts more general processing difficulty as a result of similarity between constituents as it does not make reference to c-command. Although the two theories were developed to account for different linguistic phenomena and may not always operationalize which 
features are relevant for a particular linguistic dependency in the same manner, for present purposes, both accounts highlight the role that similarity between sentence constituents plays in determining successful linguistic dependency resolution.

\section{The subject/object asymmetry in L2 processing}

The asymmetry between subject and object RCs can also inform debate about the nature of $\mathrm{L} 2$ processing. Potential L1/L2 differences in processing have been described variably in terms of shallow syntactic processing in L2 comprehension (Clahsen and Felser, 2006, 2018), reduced L2 memory capacity (McDonald, 2006) or increased susceptibility to interference in L2 learners (Cunnings, 2017). Given the memory demands of object RCs, they provide a way of examining how working memory influences L2 processing. Many approaches to working memory in L2 acquisition and processing rely on a capacitybased view that describes L1/L2 differences in terms of L2 learners lacking processing capacity. McDonald (2006), for example, explained L1/L2 differences in terms of reduced memory capacity in L2 processing. This capacity-based view claims that individuals with lower memory capacity, such as L2 learners processing their L2, have difficulty compared to higher capacity readers. Following recent advances in L1 processing that eschew the notion of memory capacity in favour of focusing on how memory encoding, storage and retrieval influence real-time sentence processing (e.g. Lewis et al., 2006; Van Dyke and Johns, 2012), Cunnings (2017) argued that L1/L2 differences can be explained in terms of L2 learners being more susceptible to memory interference during processing than L1 readers. Cunnings in particular claimed that L2 learners may weight cues to memory retrieval during processing differently to L1 readers. Cunnings' claims about interference in L2 processing were largely based on previous studies examining anaphora resolution (e.g. Felser and Cunnings, 2012), and focused on retrieval rather than encoding interference. RCs however provide a test-case to examine the role that interference may play more broadly in L2 sentence processing.

Studies have indicated that like L1 English readers, L2 English learners also find object RCs more difficult than subject RCs, as indicated by longer reading times and lower comprehension accuracy for object RCs (Baek, 2012; Street, 2017; Xia et al., 2020; see also Hopp, 2016, who examined the subject/object asymmetry in cleft constructions). Street (2017), for example, compared active sentences like (3a), to sentences containing subject and object RCs as in (3b) and (3c) respectively in a picture selection task. Participants included two groups of L1 English speakers, with either lower or higher levels of academic attainment, and a group of L2 learners with high levels of academic attainment from various L1 backgrounds.

(3) a. The girl kicked the boy in the leg.

b. This is the girl that kicked the boy.

c. This is the boy that the girl kicked.

All groups had longer reaction times for the picture selection task for sentences containing object RCs than either active sentences or subject RCs. Descriptively, all groups were at ceiling in the active and subject RC conditions, while only the high attainment L1 group was at ceiling for object RCs. Analysis of response accuracy yielded a significant 
interaction between the object $\mathrm{RC}$ condition and educational attainment but not between object RC and group (L1 vs. L2), with the lower academic attainment L1 ers having the lowest accuracy across groups in the object RC condition. Street took these results as evidence against shallow L2 processing and argued that RCs do not cause an insurmountable problem in L2 acquisition.

While such results illustrate the subject/object asymmetry in L2 comprehension, we are aware of only two studies that have examined how noun similarity influences L2 processing of the subject/object asymmetry. Though not testing RCs, Hopp (2017) examined the subject/object asymmetry in wh-questions as in $(4 \mathrm{a} / \mathrm{b})$ and $(4 \mathrm{c} / \mathrm{d})$ using the visual world paradigm. Similarity was also manipulated, such that the two nouns either mismatched $(4 \mathrm{a} / \mathrm{c})$ or matched $(4 \mathrm{~b} / \mathrm{d})$ in number. German L2 English learners' comprehension accuracy was lower for object than subject wh-questions, with the difference between object and subject questions being particularly pronounced for lower proficiency learners. High-intermediate but not advanced learners' accuracy was influenced by match in the object conditions, with lower accuracy for object RCs with two matching nouns, as in (4c), than mismatching nouns, as in (4d).

(4) a. Which cows are pushing the goat?

b. Which cow is pushing the goat?

c. Which cow are the goats pushing?

d. Which cow is the goat pushing?

Participants' gaze across a visual display illustrating the correct and incorrect interpretations of the sentences (e.g. cow/s kicking a goat and a goat kicking cow/s) indicated that learners initially interpreted the wh-phrase to be the subject of the verb in both subject and object questions. While advanced learners quickly overcame this initial misinterpretation, reanalysis was delayed for high-intermediate learners, whose reanalysis was quicker in object mismatch condition (4c) than object match condition (4d). While these findings might be suggestive of similarity-based interference, note that as number is marked on the auxiliary verb, the initial subject misinterpretation of the wh-phrase is disambiguated earlier in the number mismatch conditions. As such, these findings may result from differences in the time-course of reanalysis in the match and mismatch conditions rather than similarity-based interference.

Xia et al. (2020) examined similarity-based effects, couched within the framework of relativized minimality, in subject and object RCs. They tested constructions as in (5), which do not contain an auxiliary verb and thus avoid confounds related to reanalysis. They tested L1 English speakers and Chinese L2 English learners. Chinese L2 learners in particular were tested as although the object RC disadvantage is attested in a number of languages, Chinese arguably shows the opposite preference (for review, see Lau and Tanaka, 2021).

(5) a. I know the king who pushed the boy.

b. I know the king who pushed the boys.

c. I know the king who the boy pushed.

d. I know the king who the boys pushed. 
L1 speakers' accuracy was lower for object RCs than subject RCs but was not influenced by match/mismatch. L2 learners' accuracy was also lower for object than subject RCs, especially for lower proficiency learners, but additionally less accurate for sentences with mismatching than matching nouns, the opposite to what would be predicted by similarity-based interference. Xia et al. (2020) also did not report any significant interactions between number match and RC type, which would also be expected.

For reading times, L1 English speakers and advanced L2 learners had longer sentence reading times for object RCs than subject RCs. Neither the effect of match, nor the interaction, was significant in either L1 or L2 readers. More fine-grained analyses indicated that at the second noun in object RCs, L2 learners but not L1 readers had significantly longer reading times in mismatch than match conditions. Although this is the opposite to what would be predicted by similarity-based interference, as the match condition always included two singular nouns, interpretation of this finding is complicated by the fact that plural nouns take longer to process than singulars (Wagers et al., 2009).

In summary, the subject/object asymmetry has been observed in L2 learners in both online processing and offline comprehension measures. The one existing study that unambiguously examined similarity-based effects, Xia et al. (2020), did not find a pattern of results that would be predicted by similarity-based interference, in either L1 or L2 speakers. The fact that the L2ers' L1, Chinese, does not have obligatory number marking on nouns may have influenced the results here however. How similarity-based interference may influence L2 processing using other similarity-based manipulations, such as those used by Gordon et al. (2001, 2006), has not to our knowledge previously been examined.

\section{The present study}

Against this background, we examined similarity-based interference in subject and object RCs in L2 processing, manipulating noun similarity by comparing sentences containing two common nouns, or a common noun and a proper name (Gordon et al., 2001, 2006). We aimed to test the extent to which L1 and L2 readers are sensitive to similaritybased interference during both online processing and offline comprehension. An important issue in the $\mathrm{L} 1$ processing literature has been whether object RCs in subject position also incur processing difficulty at the subsequent matrix verb. To our knowledge, this issue has not been previously examined in the L2 processing literature. Examining processing at both the RC region and matrix verb allow us to examine how different types of similarity-based encoding and retrieval interference influence L2 processing.

\section{The study}

Participants in our study completed two tasks. In the first experiment, participants read sentences as in (6) while their eye-movements were monitored. RC type was manipulated such that $(6 \mathrm{a} / \mathrm{b})$ contain object RCs while $(6 \mathrm{c} / \mathrm{d})$ contain subject RCs. Noun similarity was also manipulated, such that the sentence subject and noun within the RC were either similar, as in $(6 \mathrm{a} / \mathrm{c})$ that contains two common nouns, or dissimilar, as in $(6 \mathrm{~b} / \mathrm{d})$ which contains a common noun and a proper name. We ensured that the RC verb ('saw') 
and matrix verb ('walked') were non-adjacent, by including spillover text ('yesterday afternoon') in between. All critical sentences were followed by a wrap-up sentence to minimize end-of-trial artefacts from influencing reading of the critical sentences. Our study design, sampling method and analysis plan were pre-registered on the Open Science Framework (OSF). Our pre-registration, materials, data and analysis code can be found at the OSF website (https://osf.io/nu25m/).

(6) a. Object RC, Similar

The boy that the girl saw yesterday afternoon, walked through the park. The weather was beautiful.

b. Object RC, Dissimilar

The boy that Rebecca saw yesterday afternoon, walked through the park. The weather was beautiful.

c. Subject RC, Similar

The boy that saw the girl yesterday afternoon, walked through the park. The weather was beautiful.

d. Subject RC, Dissimilar

The boy that saw Rebecca yesterday afternoon, walked through the park. The weather was beautiful.

We examined reading times at both the $\mathrm{RC}$ region and the matrix verb. At the $\mathrm{RC}$ region, we expected to replicate previous findings in the L1 group and find evidence of similarity-based encoding interference (Gordon et al., 2001, 2006). That is, object RCs were predicted to cause processing difficulty when the two nouns were similar, such that (6a) should have longer reading times than (6b). Similarity was not predicted to influence reading times of subject $\mathrm{RCs}$, as in $(6 \mathrm{c} / \mathrm{d})$. Thus, a crucial prediction of similarity-based encoding interference is an interaction between $\mathrm{RC}$ type and noun similarity at the RC.

If retrieval interference influences processing times at the matrix verb (Lewis and Vasishth, 2005; Van Dyke, 2007), retrieval of the sentence subject should be more difficult when a subject intervenes. If retrieval interference influences processing at the matrix verb, we should find longer reading times at the matrix verb following object RCs than subject RCs. Gordon et al. (2001, 2006) observed similarity-based encoding effects at both the RC region and main verb. If we replicate this effect in our study, and find evidence of encoding interference at both verbs, we should also observe an RC type by noun similarity interaction at the matrix verb.

Like L1 readers, we expected L2 learners to have difficulty with object RCs (Baek, 2012; Street, 2017; Xia et al., 2020). If L2 learners are more susceptible to interference than L1 speakers (Cunnings, 2017), L2 speakers should show larger interference effects. That is, during processing of the RC, L2 learners should show a larger effect of similarity-based encoding interference than L1 speakers. Specifically, the effect of similarity in object RCs should be larger in L2 learners than L1 speakers. This would crucially predict an interaction between RC type, noun similarity and group during processing of the RC. Note that increased interference in L2 learners would be difficult to explain in terms of L2 shallow parsing (Clahsen and Felser, 2006, 2018), given that the sentence structure across similar and dissimilar conditions is identical. It is also not clear how this could be 
explained in terms of L2 learners being capacity-limited (McDonald, 2006). Additionally, if L2 learners are more susceptible to retrieval interference, L2 learners should show particular difficulty at the matrix verb following object rather than subject RCs. Increased retrieval interference in L2ers in this case would be evidenced by a group by RC type interaction at the matrix verb.

Participants also completed an offline comprehension experiment in a separate session after the eye-movement experiment. This task tested the same four conditions, but tested different sentences, as in (7). Participants simply read the sentences in full, and then answered a comprehension question. The comprehension question always targeted interpretation of the matrix verb. While previous studies have included comprehension questions probing both the RC verb and matrix verb, with mixed results relating to effects of similarity-based interference (compare Gordon et al., 2001, 2006), we probed interpretation of the matrix verb only to maximize statistical power to observe effects related to this particular aspect of sentence processing. We expected object RCs to have lower accuracy than subject RCs. Object RCs with similar nouns, as in (7a), should have lower accuracy than those with dissimilar nouns, as in (7b), while noun similarity should not influence subject RCs. If L2ers are more susceptible to interference than L1ers, these effects should be larger in the L2 group.

(7) a. Object RC, Similar

The passenger that the pilot saw before the flight, seemed to be nervous.

b. Object RC, Dissimilar

The passenger that Joseph saw before the flight, seemed to be nervous.

c. Subject RC, Similar

The passenger that saw the pilot before the flight, seemed to be nervous.

d. Subject RC, Dissimilar

The passenger that saw Joseph before the flight, seemed to be nervous.

Question: Who seemed to be nervous?

The passenger / The pilot (Joseph)

\section{Participants}

Participants included 80 L1 English speakers (14 males, mean age $=21$, range $=$ 18-60) and 80 L2 English speakers (13 males, mean age $=25$, range $=18-54$ ) from the University of Reading community. The L2 speakers had different L1 backgrounds. ${ }^{1}$ The L1 participants all reported English as their only L1, while the L2 participants all reported English as their L2. The L2 participants started learning English in a school environment after age five. L2 participants completed the Quick Placement Test. Their average score was 46 (range 30-60), classing our L2 speakers as intermediate to advanced learners. Participants received course credit or were paid a small fee for taking part.

As per our pre-registration, we continued recruitment until we had 80 participants in each group, on the condition that all participants needed to score at least $70 \%$ correct on the comprehension questions in the eye-movement study, as in index of their paying attention. L2 participants also needed to score 30/60 on the proficiency test. 


\section{Materials}

Each participant completed an eye-tracking during reading task and an offline comprehension task. ${ }^{2}$ L2 participants also completed the Quick Placement Test. Experimental items in the eye-tracking experiment consisted of 24 sentences as in (6), that manipulated RC type (object vs. subject) and noun similarity (similar vs. dissimilar). All sentences included a critical sentence containing an RC and a wrap-up sentence, and the entire trial always fit on one line onscreen. Seventy-two one-line filler texts were also constructed, that contained a variety of different sentence structures. ${ }^{3}$ Participants thus read 96 texts in total in the eye-tracking experiment. Experimental and filler trials were all followed by a yes/no comprehension question to ensure participants paid attention during reading. Questions to the experimental items never probed the critical dependencies or manipulations.

There were also 96 sentences in the offline task. Experimental items included 24 sentences as in (7). The comprehension question was shown after participants read the critical sentences. Comprehension questions always probed interpretation of the matrix verb and included two answer options - the matrix verb subject (the correct answer) and the intervening noun. Seventy-two fillers were also constructed that contained different types of sentence structures, and a variety of question types, all with two options of which only one was correct.

\section{Procedure}

The study was conducted in two sessions. In the first session, participants provided informed consent and completed the eye-tracking experiment. Eye-movements were monitored using an SR Research Eyelink 1000. While viewing was binocular, the eye-movement record was recorded from the right eye only. Each session began with calibration of the eye-tracker on a 9-point grid, and recalibration was performed between trials as required. Before each trial, a gaze trigger appeared above the first word to be displayed. Upon fixation of this trigger, the text appeared. Participants were instructed to read each text at their normal reading rate, pressing a button when they were ready to move on. After reading each sentence, a yes/no comprehension question was displayed which was answered by pressing one of two buttons on a gamepad. Experimental items were pseudo-randomized with the fillers in a Latinsquare design. The first session lasted 30-45 minutes and 45-60 minutes for L1 and L2 participants respectively.

All other tasks were completed in a separate second session that was conducted at least one week after the first session. These tasks were administered via a web browser using the IbexFarm platform (https://spellout.net/ibexfarm), though completed in a lab setting. Participants first completed the offline comprehension task. They simply read each sentence at their own pace, pressing the space bar when they were ready to move on. The comprehension question then appeared on-screen, which they answered by pressing either the ' 1 ' or ' 2 ' keys for the two answer options. Across items the correct answer was counterbalanced to be the first and second answer option. The experimental and filler items were pseudo-randomized in a Latin-square design. ${ }^{4}$ The L2 participants 
then completed the Quick Placement Test. This session took approximately 30 minutes for the L1 group and 45 minutes for the L2 group.

\section{Data analysis}

For the eye-movement data, we pre-registered separate analyses at the $\mathrm{RC}$ and also the matrix verb. In the RC analysis, we analysed reading times at the RC region, which consisted of the RC (that the girl/Rebecca saw - that saw the girl/Rebecca), and a spillover region that consisted of the following words up to but not including the matrix verb ('yesterday afternoon'). In the matrix verb analysis, we analysed reading times at the matrix verb ('walked') and for spillover effects, the sentence final region, which consisted of the rest of the sentence ('through the park'). We calculated three standard eyemovement measures at each region. First-pass time sums fixations within a region before it is exited to the left or right, while regression path time sums fixations, starting when a region is first entered up until but not including, the first fixation in a region to the right. Regions that were initially skipped during reading were treated as missing data in these two measures. Total viewing time summed all fixations within a region. Regions that were not fixated at all were treated as missing data in this measure. Individual fixations that were shorter than $80 \mathrm{~ms}$ that were within one character of a neighbouring fixation were merged, while all other fixations below $80 \mathrm{~ms}$, along with those above $800 \mathrm{~ms}$, were removed before analysis. Trials with excessive track loss were also removed, accounting for less than $0.01 \%$ of the data in both groups.

Reading times were log-transformed to remove skew (Vasishth and Nicenboim, 2016) and analysis was conducted with mixed-effects models using the lme4 package (Bates et al., 2015) in R (R Core Team, 2020). In the RC analysis, fixed effects included group (L1 vs. L2), region ( $\mathrm{RC}$ region vs. spillover region), RC (object vs. subject), similarity (similar vs. dissimilar nouns) and their interactions. All fixed effects were sum coded $(-1 / 1)$, with the two levels for each fixed effect coded in the order introduced in the previous sentence (e.g. the effect of group was coded -1 for L1 and 1 for L2). Analysis of the matrix verb was similar, except region consisted of the matrix verb (coded -1) and sentence final region (coded 1). By-subject and by-item random intercepts and slopes were fit using the 'maximal' random effects structure that converged (Barr et al., 2013). As including region as a fixed effect includes two non-independent datapoints from the same trial, we also included a random intercept for trial, along with a by-trial random slope for region (see Cunnings and Sturt, 2018). If the maximal model failed to converge, we first removed the random correlation parameters. If this model still failed to converge, we iteratively removed the random effect that accounted for the least variance until convergence was achieved.

As we used sum-coding, (non-interaction) fixed effects are interpreted as main effects. To interpret interactions, we ran follow-up analyses using nested contrasts. In the case of RC by similarity interactions, nested contrasts examined similarity effects at the two levels of RC type. That is, we re-ran the mixed-effects model as in the main analysis, but replaced the main effect of similarity and the $\mathrm{RC}$ by similarity interaction terms with two sum-coded nested contrasts, one that compared the similar and dissimilar conditions in subject RCs, and one comparing the same conditions in object RCs. Other interactions 
between these variables and group and/or region were also followed-up with the appropriate sum-coded nested contrasts.

Pre-registered analysis of the comprehension task used generalized (binomial) mixedeffects models, with responses coded as correct (1) or incorrect (0). Similarly to the eyetracking data, the analysis included sum coded (-1/1) fixed effects of group (L1 vs. L2), RC (object vs. subject), similarity (similar vs. dissimilar) and their interactions. By-subject and by-item random intercepts and slopes were fit using the maximal random effects structure that converged.

\section{Results}

\section{Eye-tracking experiment}

Average accuracy to the comprehension questions was 94\% in the L1 group (range $81 \%$ - 99\%) and 93\% in the L2 group (range 71\% - 100\%), indicating that participants paid attention to the task. Reading times are reported in Table 1, while Tables 2 and 3 provide a summary of the inferential statistics at the $\mathrm{RC}$ and spillover region, and matrix verb and sentence final region, respectively. We found significant main effects of group, with longer reading times in L2 speakers in all measures, which we do not discuss further. We also do not discuss main effects of region or the group by region interaction below, as these effects on their own, unless they further interact with RC type or noun similarity, are unrelated to our research questions.

a RC analysis. First-pass times in the RC analysis revealed a significant main effect of similarity (estimate $=0.016, S E=0.01, t=2.05, p=.041$ ) with longer reading times in similar than dissimilar conditions, that was modulated by a significant region by similarity interaction (estimate $=-0.017, S E=0.01, t=-2.37, p=.018$ ). Nested contrasts indicated a significant similarity effect, with longer reading times in the similar than the dissimilar condition at the $\mathrm{RC}$ region (estimate $=0.033, S E=0.01, t=2.58, p=.010$ ) but not the spillover region (estimate $=-0.001, S E=0.01, t=-0.11, p=.909$ ). Although this effect was numerically larger in the L1 group, the group by region by distractor interaction in the main analysis was not significant. Note that this main effect of similarity may be related to the different lexical material tested across the similar and dissimilar conditions, and as such we do not discuss it further.

Regression path times at the RC and spillover regions are illustrated in Figure 1. Analysis revealed a significant group by region by $\mathrm{RC}$ interaction (estimate $=0.017, \mathrm{SE}$ $=0.01, t=2.49, p=.013$ ), indicating that subject and object RCs differed by group and region. To examine this interaction, we conducted nested contrasts examining RC effects for each group at each region. These indicated that the effect of $\mathrm{RC}$ was not significant in either group at the $\mathrm{RC}$ region (both estimates $<0.020, S E \mathrm{~s}<0.03, t \mathrm{~s}<1.64$. $p \mathrm{~s}>$ .102), however at the spillover region, the effect of RC was significant in the L1 group only, though subject RCs had longer reading times overall than object RCs (L1 estimate $=-0.030, S E=0.02, t=-1.97, p=.049 ;$ L2 estimate $=0.007, S E=0.02, t=0.36, p$ $=.722$ ). In the main analysis, there was also a significant main effect of similarity (estimate $=0.034, S E=0.01, t=4.47, p<.001)$ that was modulated by a significant 


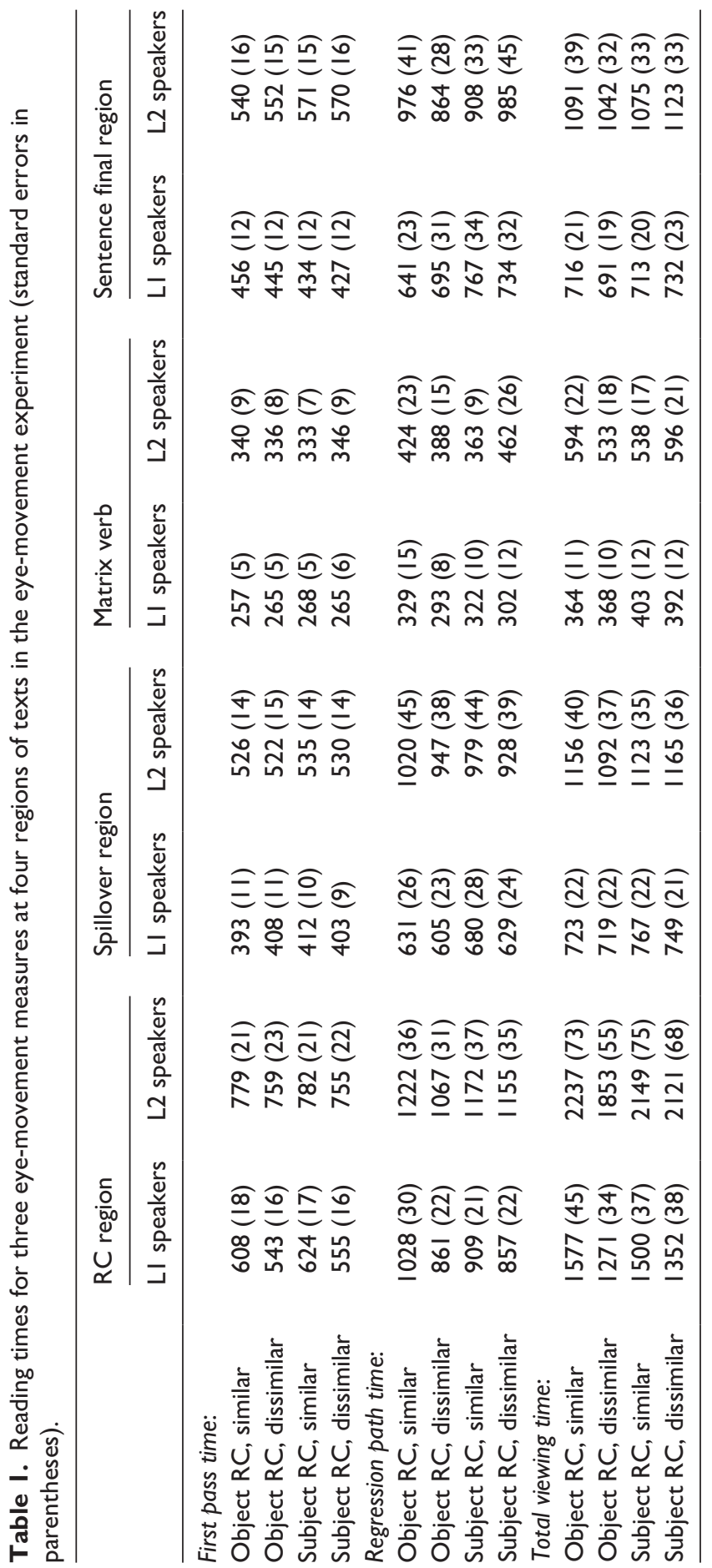




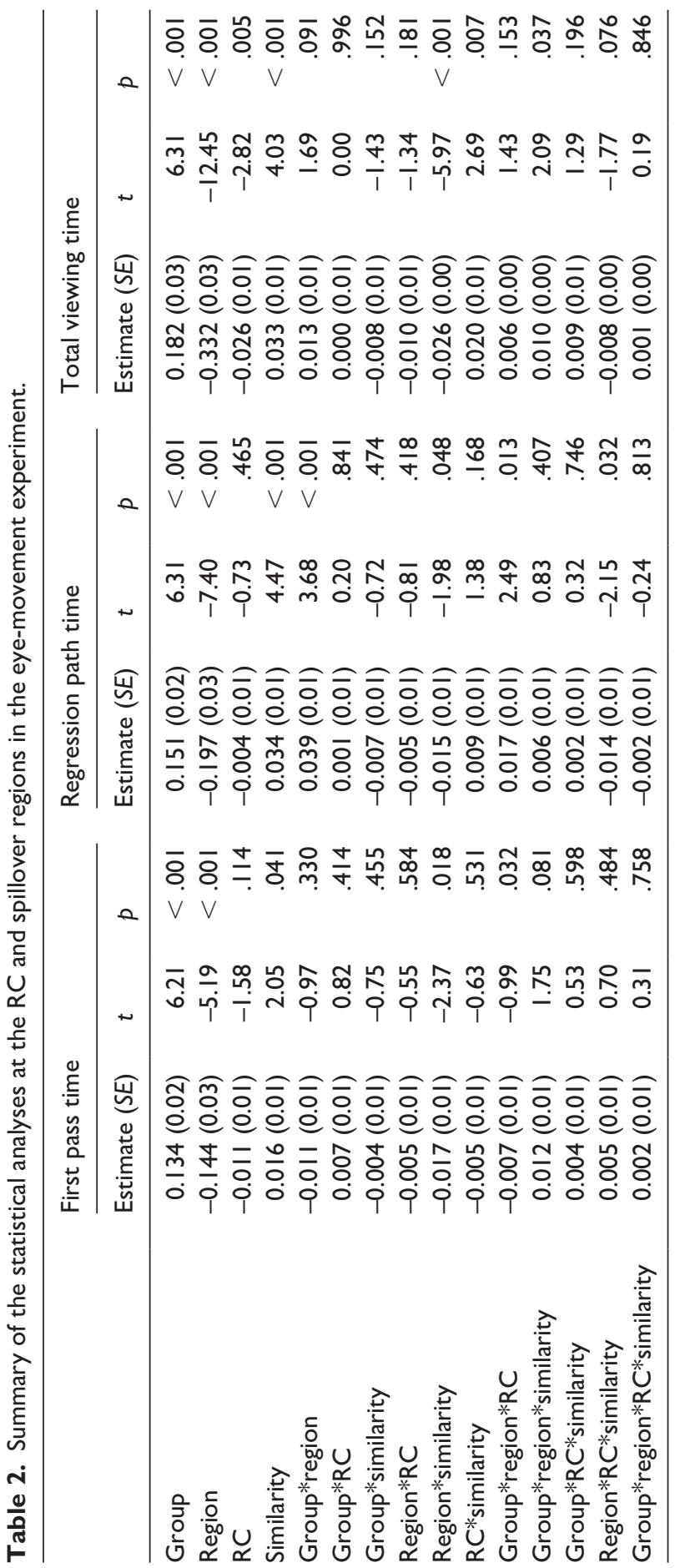




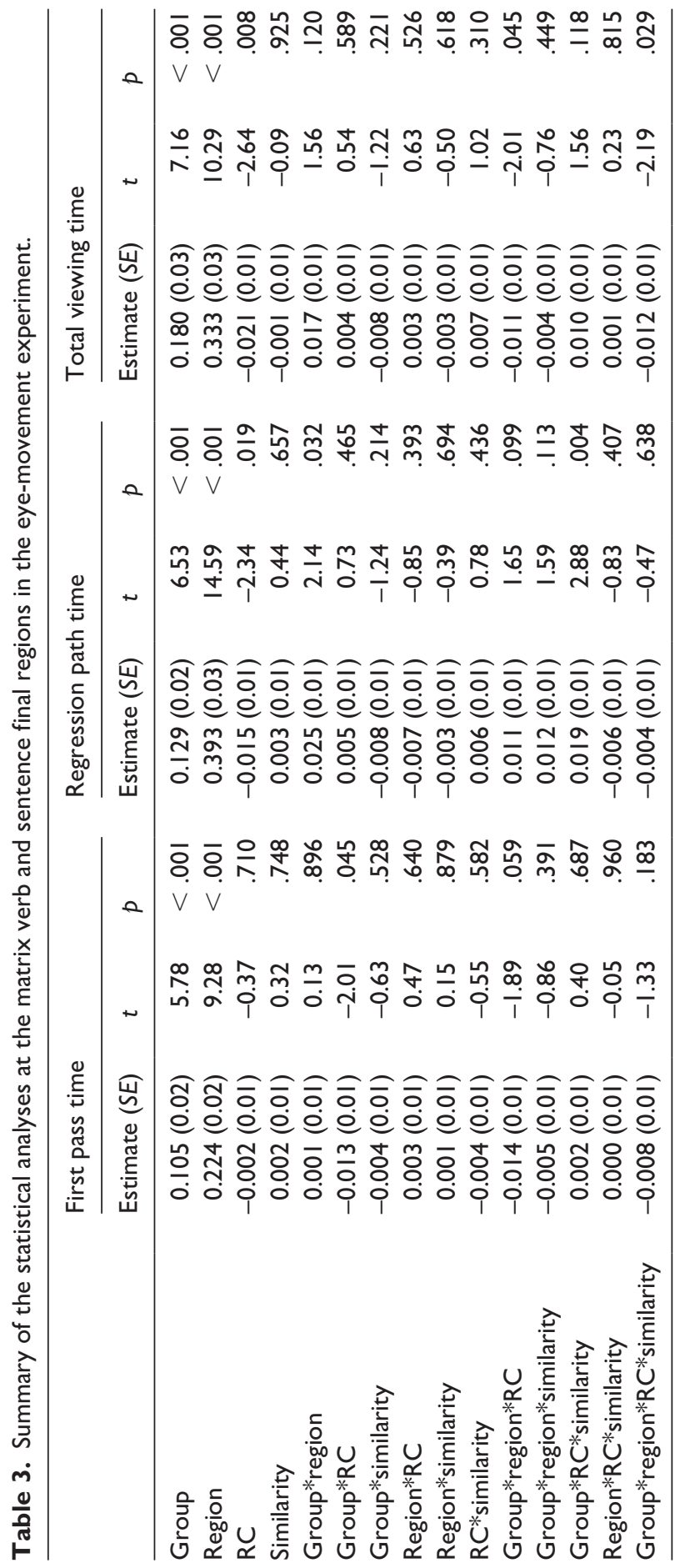




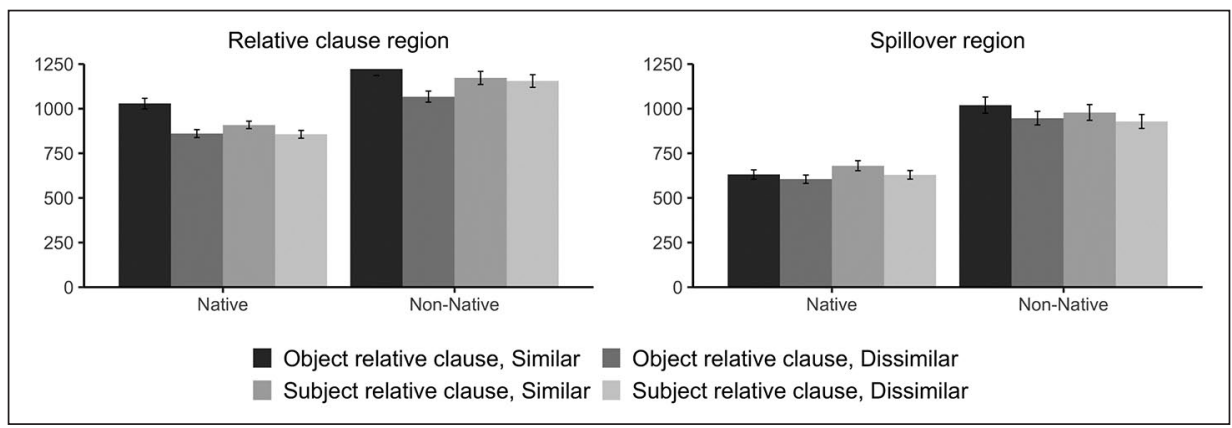

Figure I. Regression path times in milliseconds at the relative clause and spillover regions.

Note. Error bars indicate standard errors.

two-way interaction between region and similarity (estimate $=-0.015, S E=0.01, t=$ $-1.98, p=.048$ ) and, crucially, a significant three-way interaction between region, $\mathrm{RC}$ and similarity (estimate $=-0.014, S E=0.01, t=-2.15, p=.032$ ). As none of the relevant interactions between these variables and group were significant, to examine the three-way interaction, we conducted nested contrasts, collapsed across groups, comparing similarity effects at the two levels of $\mathrm{RC}$, at both regions of text. At the $\mathrm{RC}$ region, object RCs had significantly longer reading times in the similar condition than the dissimilar condition (estimate $=0.076, S E=0.01, t=5.03, p<.001)$. This difference was also significant in subject RCs, though the estimate was smaller in size (estimate = $0.026, S E=0.01, t=2.20, p=.028)$. Neither comparison was significant at the spillover region (both estimates $<0.025, S E \mathrm{~s}<0.03$, $t \mathrm{~s}<1.53, p \mathrm{~s}>.129$ ). The larger effect of similarity in object than subject RCs at the RC region indicates similarity-based encoding interference.

In total viewing times, there was a significant main effect of similarity (estimate = 0.033, $S E=0.01, t=4.03, p<.001$ ), a significant region by similarity interaction (estimate $=-0.026, S E<0.01, t=-5.97, p<.001)$ and a significant three-way interaction between group, region and similarity (estimate $=0.010, S E<0.01, t=2.09, p=.037$ ). To examine the three-way interaction, nested contrasts examined similarity effects for each group at each region. These indicated significantly longer reading times in similar than dissimilar conditions at the $\mathrm{RC}$ region in both groups, though the effect size was larger for the L1 speakers (L1 estimate $=0.080, S E=0.01, t=7.23, p<.001$; L2 estimate $=0.039, S E=0.01, t=3.03, p=.002)$. The same contrasts at the spillover region were however not significant (both estimates 0.009, $S E \mathrm{~s}<0.02, t \mathrm{~s}<0.57$, $p \mathrm{~s}>.577$ ). Note that, as in first-pass times, these main effects of similarity on their own are difficult to interpret due to differences in lexical material across similar and dissimilar conditions. Importantly, in the main analysis, in addition to the aforementioned main effect of similarity, there was also a significant main effect of RC (estimate $=-0.026, S E=0.01, t=$ $-2.82, p=.005$ ) and a significant RC by similarity interaction (estimate $=0.020, S E=$ $0.01, t=2.69, p=.007)$. As these effects did not significantly interact with group or region, to examine the $\mathrm{RC}$ by similarity interaction we conducted nested contrasts 


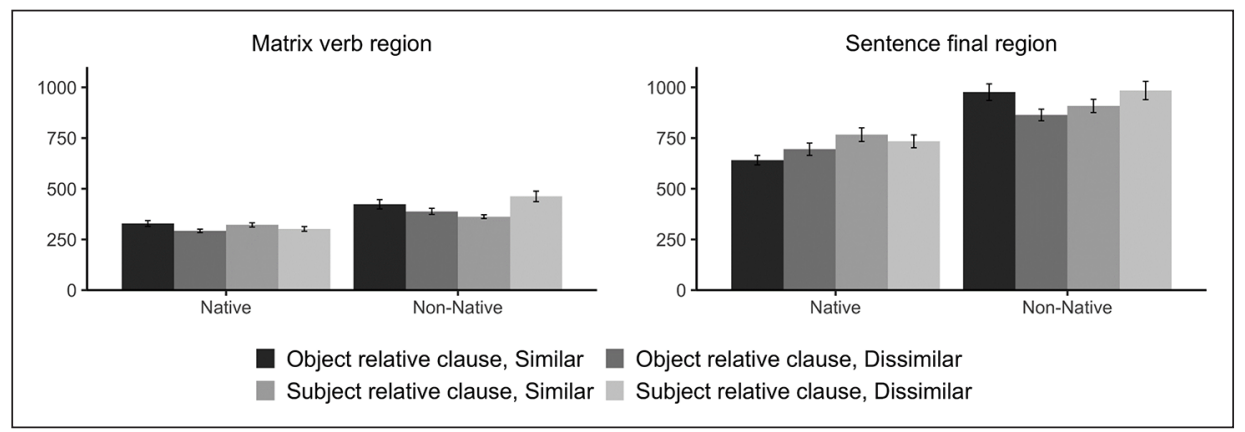

Figure 2. Regression path times in milliseconds at the matrix verb and sentence final regions. Note. Error bars indicate standard errors.

examining similarity effects in object and subject RCs, collapsed across region and group. These revealed significantly longer reading times in the similar condition than the dissimilar condition in object RCs (estimate $=0.053, S E=0.01, t=4.42, p<.001$ ), but not subject RCs (estimate $=0.013, S E=0.01, t=1.28, p=.200$ ). This effect of similarity in object but not subject RCs indicates similarity-based encoding interference. As in regression path times, this interaction was largely driven by effects at the RC region rather than the spillover region, though the three-way interaction between $\mathrm{RC}$, similarity and region in the main analysis was not significant in total viewing times.

b Matrix verb analysis. First-pass times in the matrix verb analysis revealed a significant group by RC interaction (estimate $=0.013, S E=0.01, t=-2.01, p=.045$ ), however follow-up nested contrasts indicated no significant RC effects in either group (both estimates $<0.017, S E \mathrm{~s}<0.02$, $t \mathrm{~s}<1.49, p \mathrm{~s}>.137$ ). In the main analysis, there was also a trend for a group by region by $\mathrm{RC}$ interaction but this was not significant.

Regression path times at the matrix verb and sentence final regions are shown in Figure 2. Analysis revealed a significant main effect of RC (estimate $=-0.015, S E=$ $0.01, t=-2.34, p=.019)$ that tended to differ by group and region, though the group by region by $\mathrm{RC}$ interaction was not significant. The main effect of $\mathrm{RC}$ was however modulated by a significant three-way interaction between group, $\mathrm{RC}$ and similarity (estimate $=0.019, S E=0.01, t=2.88, p=.004)$. To examine this interaction, we conducted nested contrasts, collapsed across regions, examining similarity effects for each group separately in subject and object RCs. These revealed no significant differences between the two object RC conditions in either group (both estimates $<0.021$, SEs $<0.02$, ts $<$ $1.43, p \mathrm{~s}>.154)$. There were no significant differences between the subject RC conditions in the L1 group (estimate $=-0.024, S E=0.02, t=-1.48, p=.140$ ), while for the L2 group the dissimilar condition had significantly longer reading times than the similar condition (estimate $=0.029, S E=0.01, t=2.26, p=.024$ ).

In total viewing times, there was a significant main effect of RC (estimate $=-0.021$, $S E=0.01, t=-2.64, p=.008$ ), that was modulated by a significant three-way interaction between group, region and $\mathrm{RC}$ (estimate $=-0.011, S E=0.01, t=-2.01, p=.045$ ), 
Table 4. Accuracy in percentages in the comprehension experiment (standard errors in parentheses).

\begin{tabular}{lll}
\hline & LI speakers & L2 speakers \\
\hline Object RC, similar & $79(2)$ & $89(\mathrm{I})$ \\
Object RC, dissimilar & $83(2)$ & $92(\mathrm{I})$ \\
Subject RC, similar & $82(2)$ & $94(\mathrm{I})$ \\
Subject RC, dissimilar & $81(2)$ & $92(\mathrm{I})$ \\
\hline
\end{tabular}

Table 5. Statistical analysis of the comprehension experiment.

\begin{tabular}{lrrr}
\hline & Estimate $(\mathrm{SE})$ & \multicolumn{1}{c}{$\mathrm{t}$} & \multicolumn{1}{c}{$\mathrm{P}$} \\
\hline Group & $0.524(0.08)$ & 6.34 & $<.001$ \\
RC & $-0.098(0.06)$ & -1.57 & .118 \\
Similarity & $-0.019(0.05)$ & -0.37 & .714 \\
Group*RC & $-0.082(0.07)$ & -1.24 & .215 \\
Group*similarity & $0.007(0.05)$ & 0.14 & .888 \\
RC*similarity & $-0.152(0.05)$ & -2.81 & .005 \\
Group*RC*similarity & $-0.064(0.06)$ & -1.12 & .261 \\
\hline
\end{tabular}

and a significant four-way interaction between group, region, $\mathrm{RC}$ and similarity (estimate $=-0.012, S E=0.01, t=-2.19, p=.029)$. To examine the four-way interaction, nested contrasts examined similarity effects in subject and object RCs separately for each group and region. However, these analyses revealed no significant differences between the two object RC conditions or the two subject RC conditions at either the matrix verb or sentence final region in either group (all estimates $<0.031, S E \mathrm{~s}<0.03$, $t \mathrm{~s}<1.60, p \mathrm{~s}>.113)$.

\section{Comprehension experiment}

Average comprehension accuracy rates and the inferential statistics for the comprehension experiment are provided in Tables 4 and 5 respectively. Analysis revealed a significant main effect of group (estimate $=0.524, S E=0.08, z=6.34, p<.001$ ), with the L2 group being overall more accurate than the L1 speakers. There was also a significant $\mathrm{RC}$ by similarity interaction (estimate $=-0.152, S E=0.05, t=-2.81, p=.005$ ) that did not significantly interact with group. Nested contrasts compared the two subject and two object RC conditions. These revealed that for object RCs the similar condition yielded significantly lower accuracy rates than the dissimilar condition (estimate = $-0.171, S E=0.07, t=-2.41, p=.016$ ). Subjects RCs numerically showed the opposite pattern, though the difference was not significant (estimate $=0.131, S E=0.08$, $t=1.66, p=.097)$. Though the effect of similarity was significant in the object RC conditions, we note that numerically this effect is very small. 


\section{Summary}

In sum, at the $\mathrm{RC}$ region we found significant interactions between $\mathrm{RC}$ and similarity in regression path times and total viewing times, indicating similarity-based encoding interference in object RCs in both groups. Reading times at the matrix verb were not generally longer following object RCs than subject RCs, and the significant interactions we observed between RC, similarity and group were not in the predicted direction to provide evidence of similarity-based interference. We did however observe evidence of similarity-based interference in our comprehension experiment. We discuss the implications of these findings in detail below.

\section{General discussion}

In this study, we examined how similarity-based interference influences L2 sentence processing. We found evidence of interference in both L1 and L2 processing, though we did not find evidence of increased interference in L2 learners. We discuss our results in terms of the time-course of interference in L1 and L2 sentence processing, along with a comparison of previous relevant L2 studies on RC comprehension, in turn below.

\section{Interference in LI and L2 processing}

The interactions that we observed between RC type and noun similarity at the RC region in regression path times and total viewing times support previous evidence of similaritybased interference during L1 processing (Gordon et al., 2001, 2006). In both measures, reading times were longer for object RCs that contained two common nouns rather than a common noun and proper name, while this similarity-based effect was reduced, or nonsignificant, in subject RCs. These effects at the RC region did not significantly interact with group, suggesting similarity-based interference in both L1 and L2 processing. These findings extend previous L1 results to L2 comprehension. We interpret these findings as indicating encoding interference, such that sentence constituents that are similar along a particular dimension, in this case noun type, are encoded in memory with less distinct representations, in comparison to two nouns of different types, which in turn causes processing disruption.

This effect in both groups was largely restricted to the RC region itself during processing. This was especially the case in regression path times, where interference was observed at the RC region, but did not extend to the spillover region. More importantly, although we found evidence of similarity-based interference in the analysis of the $\mathrm{RC}$, this was not observed in our analysis at the matrix verb in any measure. This contrasts with previous L1 studies (Gordon et al., 2001, 2006), where significant interactions between RC type and noun similarity were found both at the RC and the matrix verb. One difference between the current study and Gordon et al. is that we included spillover such that the RC verb (in object RCs) and matrix verb were not adjacent, while Gordon et al. did not. As such, it is difficult to be sure if the similarity-based effects reported at the matrix verb by Gordon et al. reflect difficulty at the matrix verb itself, or spillover from the $\mathrm{RC}$ region. ${ }^{5}$ 
Although we did not find evidence of similarity-based encoding interference at the matrix verb during processing, we did find some evidence of this type of interference in comprehension accuracy in our offline comprehension experiment. Here, again for both L1 and L2 groups, accuracy was lower for object RCs with similar than dissimilar nouns, but accuracy for subject RCs did not differ significantly. As our comprehension task tapped interpretation of the subject of the matrix verb, this presumably reflects encoding interference at the matrix verb, albeit in an offline rather than online measure. Note however that numerically the effect here was very small. Further research is required to assess the extent to which encoding interference influences processing at the matrix verb following an RC in L1 and L2 comprehension. Note also that while our comprehension questions focused on the matrix verb, other studies have used a mix of questions probing both the RC and matrix verbs (e.g. Gordon et al., 2001, 2006). Further research is again required here to examine comprehension of both the RC and matrix verbs in L1 speakers and L2 learners.

In addition to similarity-based encoding interference, we also considered the possibility of retrieval interference influencing processing. The most obvious evidence of this would have been at the matrix verb, where cue-based parsing would predict difficulty following object RCs, as in object RCs but not subject RCs the intervening noun is also a subject, which should cause interference (Lewis and Vasishth, 2005; Van Dyke, 2007). If L2 learners are more susceptible to interference than L1 speakers (Cunnings, 2017), this should especially be the case in our L2 group. However, neither group exhibited significantly longer reading times at the matrix verb following object as compared to subject RCs in any measure. The extent to which object RCs cause processing difficulty at the matrix verb in L1 processing has however been debated. Staub et al. (2017) claimed that these effects in L1 processing are often confounded with spillover, as they found no significant difficulty at the matrix verb when they included spillover text between the RC and matrix verb. Our results are compatible with this claim in both L1 and L2 comprehension.

One difference between our experiment and previous studies is that we included a comma to delineate between the RC and matrix verb. We are unsure about how this could have influenced our results, given that the comma appeared in all conditions, but it is at least conceivable that this may have impacted processing at the matrix verb, and may have influenced the (lack of) effects of RC type here. Note however that our null effects of RC type at the matrix verb are similar to studies that have included spillover between the RC and matrix verb, which also failed to find significant effects of RC type at the matrix verb (Grodner and Gibson, 2005; Staub et al., 2017). Further research is required here to assess how punctuation may influence processing of sentences containing RCs.

The one significant difference between L1 and L2 processing that we did observe was however found at the matrix verb. This was clearest in regression path times. Here, collapsing across the matrix verb and sentence final regions, neither the L1 nor L2 groups showed significant differences in the two object RC conditions. L1 readers also did not show any significant differences between the two subject RC conditions, while L2 learners had significantly longer reading times for subject RCs in the dissimilar condition than the similar condition. This effect in subject RCs in the L2 readers was unexpected. Note however that it is not in the correct direction to suggest increased interference in L2 learners. It is also not clear how this pattern could be explained in terms of L2 shallow 
processing or reduced L2 processing capacity. One potential account could be that it indexes L2 difficulty in retrieving the sentence subject at the matrix verb when a proper name intervenes, due to the discourse salience of proper names over common nouns (Sanford et al., 1988). However, in that case, it is unclear why this should influence sentences containing subject RCs but not object RCs. Indeed, if discourse salience of the intervening noun matters, we might expect this effect to be bigger in sentences containing object RCs, given the intervening proper name is also a subject in object but not subject RCs, but the opposite pattern was observed. For these reasons, we do not draw any strong conclusions about what this effect for L2 learners may index and leave it to future research to assess the replicability of this unexpected finding.

In summary, although we did not find significant evidence of increased interference in L2 learners (see Cunnings, 2017), our results indicate that similarity-based encoding interference influences RC processing in both $\mathrm{L} 1$ and $\mathrm{L} 2$ comprehension.

\section{A comparison to previous $L 2$ studies}

Previous L2 research has indicated lower comprehension accuracy in object than subject RCs (e.g. Street, 2017; Xia et al., 2020; for similar results in wh- and cleft constructions, see also Hopp, 2016, 2017). Although we found evidence of similarity-based interference in comprehension accuracy in sentences containing object RCs, sentences containing object RCs as a whole were not comprehended less accurately than sentences with subject RCs. Note here that our comprehension question tapped understanding of the matrix verb, while previous studies tested accuracy of the RC itself. Thus, our comprehension results are not directly comparable to previous studies.

In his study of subject and object wh-questions, Hopp (2017) reported that high-intermediate L2 learners had particular difficulty with object wh-questions in sentences containing two number matching rather than mismatching nouns. As discussed in the Introduction, whilst this might suggest similarity-based interference, given that the temporary ambiguity of wh-questions was disambiguated earlier in the number mismatch conditions, Hopp's results may also be explained by the time-course of reanalysis. The reading time data we observed at the $\mathrm{RC}$ region provide clearer support for similaritybased interference in $\mathrm{L} 2$ processing.

Most relevant to the current study are the self-paced reading results reported by Xia et al. (2020), who did not observe evidence of similarity-based interference in their study manipulating number (mis)match in RCs in Chinese L2 learners of English. Indeed, dissimilar conditions caused difficulty in comparison to similar conditions in Xia et al.'s L2 data. There are a number of differences between our study and Xia et al. that may explain these findings. One difference is that Xia et al. tested Chinese L2 learners, whose L1 both does not contain overt number morphology on nouns. This potentially may have influenced the L2 learners' sensitivity to Xia et al.'s number (mis)match manipulation. Chinese is also argued to show an object rather than subject RC preference. Either factor may contribute to the different findings between our study and Xia et al.'s results.

To examine the possibility of L1 background influencing our results, we conducted additional, exploratory (non-preregistered) analyses of our eye-movement data examining whether the L2 learners' L1 background influenced our results. In research examining L2 processing of object gap dependencies, whether an L2 learners' L1 allows 
wh-movement or not has been a topic of debate (e.g. Kim et al., 2015). Given that English is a wh-movement language, the hypothesis here would be that L2 learners' whose L1 allows wh-movement may be more nativelike than L2 learners with a wh-in-situ L1. Our 80 L2 participants were equally divided into two groups of 40 L2 learners with a whmovement L1 (L1 French, Greek, Bulgarian, Spanish, Romanian, German) and 40 L2 learners with a wh-in-situ L1 (Chinese, Japanese, Korean). However, in an analysis at the $\mathrm{RC}$ region, $\mathrm{L} 1$ background did not significantly modulate the crucial $\mathrm{RC}$ by similarity interactions that we observed, nor did we find any significant interactions with L1 background in analyses at the matrix verb. ${ }^{6}$ Overall differences in L2 proficiency may also influence L2 processing. For this reason, we also conducted exploratory analyses examining whether individual differences in L2 proficiency influenced our eye-movement results, but did not find consistent effects. ${ }^{7}$ As such, while we acknowledge that our L2 group is more heterogeneous than those tested by Xia et al., further research that directly compares L2 learners with different L1 backgrounds is required to fully explore potential influences of L1 background on L2 RC processing.

\section{Conclusions}

We observed similarity-based encoding interference during the processing of object RCs in L1 and L2 readers. While we did not find evidence that L2 learners are more susceptible to interference than L1 readers during processing (see Cunnings, 2017), the similarity-based effects that we observed indicate the importance of considering working memory in L1 and L2 comprehension. Many accounts of working memory in L2 comprehension focus on memory capacity (McDonald, 2006). The similarity-based effects we report are difficult to describe in terms of capacity but highlight how considerations of how representations of sentence constituents are encoded in memory can inform our understanding of L1 and L2 sentence processing.

\section{Acknowledgements}

We thank the editor and reviewers for comments on previous drafts of the manuscript.

\section{Declaration of conflicting interests}

The author(s) declared no potential conflicts of interest with respect to the research, authorship, and/or publication of this article.

\section{Funding}

The author(s) disclosed receipt of the following financial support for the research, authorship, and/ or publication of this article: The research reported here was funded by a Leverhulme Trust Research Project Grant (RPG-2018-337) awarded to IC.

\section{ORCID iDs}

Ian Cunnings (iD) https://orcid.org/0000-0002-5318-0186

Hiroki Fujita (iD https://orcid.org/0000-0001-7649-9707 


\section{Supplemental material}

Supplemental material for this article is available online.

\section{Notes}

1. First languages of the L2 participants were Chinese (25), Japanese (8), Korean (7), French (16), Greek (8), Bulgarian (4), Spanish (6), Romanian (3) and German (3).

2. Participants also completed a lexical decision task. This was administered to examine how lexical automaticity may influence L2 processing (Hopp, 2014). Additional exploratory analyses with individual differences were mainly planned if we observed significant L1/L2 differences. As we did not observe clear L1/L2 differences, we do not report analyses with lexical automaticity as an independent variable.

3. Twenty-four fillers in both the eye-tracking and offline comprehension task included experimental items from an unrelated manipulation not reported here.

4. Due to technical error, the Latin-square for the L1 data was not fully balanced, with $27,14,25$ and 14 participants completing lists $1-4$ respectively. For the L2 data, each list was completed by 20 participants.

5. Note that in regression path times we did not observe any significant similarity-based effects at the spillover region that could be considered spillover, and instead effects were restricted to the critical relative clause region. We do not speculate over why we observed very localized effects during processing of the relative clause itself, while other studies (Gordon et al., 2001, 2006) have observed effects in subsequent regions as well. The point is that when effects after the relative clause region are observed, if there is no text between the relative clause verb and matrix verb, it is difficult to tease apart whether such effects at the matrix verb index difficulty caused at the verb itself, or spillover from the relative clause.

6. Chinese L2 learners may behave differently to other L2 groups given that Chinese, but not other wh-in-situ languages, has been argued to show an object RC preference (for review, see Lau and Tanaka, 2021). However, given the small samples that would be involved in further dividing our L2 learners, we did not conduct this analysis.

7. For further details of these additional analyses, see the Supplementary Analysis document on the OSF website (https://osf.io/nu25m).

\section{References}

Baek S (2012) Processing of English relative clauses by adult L2 learners. Unpublished doctoral dissertation, University of Illinois, Urbana-Champaign, IL, USA.

Barr DJ, Levy R, Scheepers C, and Tily HJ (2013) Random effects structure for confirmatory hypothesis testing: Keep it maximal. Journal of Memory and Language 68: 255-78.

Bates D, Mächler M, Bolker B, and Walker S (2015) Fitting linear mixed-effects models using Ime4. Journal of Statistical Software 67: 1-48.

Belletti A and Rizzi L (2013) Intervention in grammar and processing. In: Caponigro I and Cecchetto C (eds) From grammar to meaning. Cambridge: Cambridge University Press, 294-311.

Clahsen $\mathrm{H}$ and Felser C (2006) Continuity and shallow structures in language processing. Applied Psycholinguistics 27: 107-26.

Clahsen H and Felser C (2018) Some notes on the shallow structure hypothesis. Studies in Second Language Acquisition 40: 693-706.

Cunnings I (2017) Parsing and working memory in bilingual sentence processing. Bilingualism: Language and Cognition 20: 659-78. 
Cunnings I and Sturt P (2018) Retrieval interference and semantic interpretation. Journal of Memory and Language 102: 16-27.

Doughty C (1991) Second language instruction does make a difference: Evidence from an empirical study of SL relativization. Studies in Second Language Acquisition 13: 431-69.

Eckman FR, Bell L, and Nelson D (1988) On the generalization of relative clause instruction in the acquisition of English as a second language. Applied Linguistics 9: 1-20.

Felser C and Cunnings I (2012) Processing reflexives in a second language: The timing of structural and discourse-level constraints. Applied Psycholinguistics 33: 571-603.

Frazier L and Clifton C (1989) Successive cyclicity in the grammar and the parser. Language and Cognitive Processes 4: 93-126.

Gas S (1979) Language transfer and universal grammatical relations. Language Learning 29: 327-44.

Gennari SP and MacDonald MC (2008) Semantic indeterminacy in object relative clauses. Journal of Memory and Language 58: 161-87.

Gibson E (1998) Linguistic complexity: Locality of syntactic dependencies. Cognition 68: 1-76.

Gordon PC and Lowder MW (2012) Complex sentence processing: A review of theoretical perspectives on the comprehension of relative clauses: Review of complex sentence processing. Language and Linguistics Compass 6: 403-15.

Gordon PC, Hendrick R, and Johnson M (2001) Memory interference during language processing. Journal of Experimental Psychology: Learning, Memory, and Cognition 27: 1411-23.

Gordon PC, Hendrick R, and Johnson M (2004) Effects of noun phrase type on sentence complexity. Journal of Memory and Language 51: 97-114.

Gordon PC, Hendrick R, Johnson M, and Lee Y (2006) Similarity-based interference during language comprehension: Evidence from eye tracking during reading. Journal of Experimental Psychology: Learning, Memory, and Cognition 32: 1304-21.

Grodner D and Gibson E (2005) Consequences of the serial nature of linguistic input for sentenial complexity. Cognitive Science 29: 261-90.

Hopp H (2014) Working memory effects in the L2 processing of ambiguous relative clauses. Language Acquisition 21: 250-78.

Hopp H (2016) The timing of lexical and syntactic processes in second language sentence comprehension. Applied Psycholinguistics 37: 1253-80.

Hopp H (2017) The processing of English which-questions in adult L2 learners: Effects of L1 transfer and proficiency. Zeitschrift für Sprachwissenschaft 36: 107-34.

Jackson CN and Roberts L (2010) Animacy affects the processing of subject-object ambiguities in the second language: Evidence from self-paced reading with German second language learners of Dutch. Applied Psycholinguistics 31: 671-91.

Johnson ML, Lowder MW, and Gordon PC (2011) The sentence-composition effect: Processing of complex sentences depends on the configuration of common noun phrases versus unusual noun phrases. Journal of Experimental Psychology: General 140: 707-24.

Kim E, Baek S, and Tremblay A (2015) The role of island constraints in second language sentence processing. Language Acquisition 22: 384-416.

King J and Just MA (1991) Individual differences in syntactic processing: The role of working memory. Journal of Memory and Language 30: 580-602.

Lau E and Tanaka N (2021) The subject advantage in relative clauses: A review. Glossa: A Journal of General Linguistics 6: 34.

Levy R (2008) Expectation-based syntactic comprehension. Cognition 106: 1126-77.

Lewis RL and Vasishth S (2005) An activation-based model of sentence processing as skilled memory retrieval. Cognitive Science 29: 375-419. 
Lewis RL, Vasishth S, and Van Dyke JA (2006) Computational principles of working memory in sentence comprehension. Trends in Cognitive Sciences 10: 447-54.

Lowder MW and Gordon PC (2012) The pistol that injured the cowboy: Difficulty with inanimate subject-verb integration is reduced by structural separation. Journal of Memory and Language 66: 819-32.

Lowder MW and Gordon PC (2014) Effects of animacy and noun-phrase relatedness on the processing of complex sentences. Memory and Cognition 42: 794-805.

McDonald JL (2006) Beyond the critical period: Processing-based explanations for poor grammaticality judgment performance by late second language learners. Journal of Memory and Language 55: 381-401.

R Core Team (2020) R: A language and environment for statistical computing [software]. Vienna: R Foundation for Statistical Computing. Available at: https://www.R-project.org (accessed November 2021).

Reali F and Christiansen MH (2007) Processing of relative clauses is made easier by frequency of occurrence. Journal of Memory and Language 57: 1-23.

Rizzi L (1990) Relativized minimality. Cambridge, MA: MIT Press.

Rizzi L (2018) Intervention effects in grammar and language acquisition. Probus 30: 339-67.

Roland D, Dick F, and Elman JL (2007) Frequency of basic English grammatical structures: A corpus analysis. Journal of Memory and Language 57: 348-79.

Sanford AJ, Moar K, and Garrod SC (1988) Proper names as controllers of discourse focus. Language and Speech 31: 43-56.

Staub A (2010) Eye movements and processing difficulty in object relative clauses. Cognition 116: 71-86.

Staub A, Dillon B, and Clifton C (2017) The matrix verb as a source of comprehension difficulty in object relative sentences. Cognitive Science 41: 1353-76.

Street JA (2017) This is the native speaker that the non-native speaker outperformed: Individual, education-related differences in the processing and interpretation of Object Relative Clauses by native and non-native speakers of English. Language Sciences 59: 192-203.

Traxler MJ, Morris RK, and Seely RE (2002) Processing subject and object relative clauses: Evidence from eye movements. Journal of Memory and Language 47: 69-90.

Traxler MJ, Williams RS, Blozis SA, and Morris RK (2005) Working memory, animacy, and verb class in the processing of relative clauses. Journal of Memory and Language 53: 204-24.

Van Dyke JA (2007) Interference effects from grammatically unavailable constituents during sentence processing. Journal of Experimental Psychology: Learning, Memory, and Cognition 33: 407-30.

Van Dyke JA and Johns CL (2012) Memory interference as a determinant of language comprehension: Interference in comprehension. Language and Linguistics Compass 6: 193-211.

Vasishth S and Nicenboim B (2016) Statistical methods for linguistic research: Foundational ideas - Part I. Language and Linguistics Compass 10: 349-69.

Wagers MW, Lau EF, and Phillips C (2009) Agreement attraction in comprehension: Representations and processes. Journal of Memory and Language 61: 206-37.

Wells J, Christiansen M, Race D, Acheson D, and Macdonald M (2009) Experience and sentence processing: Statistical learning and relative clause comprehension. Cognitive Psychology 58: 250-71.

Xia VY, White L, and Guzzo NB (2020) Intervention in relative clauses: Effects of relativized minimality on L2 representation and processing. Second Language Research. Epub ahead of print 20 September 2020. DOI: 10.1177/0267658320958742. 